\title{
Fingermark initial composition and aging using Fourier transform infrared microscopy
} ( $\mu$-FTIR)

\author{
Aline Girod ${ }^{\mathrm{a}}$, Linda Xiao ${ }^{\mathrm{b}}$, Brian Reedy $^{\mathrm{b}}$, Claude Roux ${ }^{\mathrm{b}}$, Céline Weyermann ${ }^{\mathrm{a}}$ \\ ${ }^{a}$ Institut de Police Scientifique, University of Lausanne, Bâtochime, CH -1015 Lausanne, Switzerland \\ ${ }^{\mathrm{b}}$ Centre for Forensic Science, University of Technology, Sydney, Broadway NSW 2007, Australia
}

\begin{abstract}
This study investigated fingermark residues using Fourier transform infrared microscopy ( $\mu$ FTIR) in order to obtain fundamental information about the marks' initial composition and aging kinetics. This knowledge would be an asset for fundamental research on fingermarks, such as for dating purposes. Attenuated Total Reflection (ATR) and single-point reflection modes were tested on fresh fingermarks. ATR proved to be better suited and this mode was subsequently selected for further aging studies. Eccrine and sebaceous material was found in fresh and aged fingermarks and the spectral regions $1000-1850 \mathrm{~cm}^{-1}$ and $2700-3600 \mathrm{~cm}^{-1}$ were identified as the most informative. The impact of substrates (aluminium and glass slides) and storage conditions (storage in the light and in the dark) on fingermark aging was also studied. Chemometric analyses showed that fingermarks could be grouped according to their age regardless of the substrate when they were stored in an open box kept in an air-conditioned laboratory at around $20^{\circ} \mathrm{C}$ next to a window. On the contrary, when fingermarks were stored in the dark, only specimens deposited on the same substrate could be grouped by age. Thus, the substrate appeared to influence aging of fingermarks in the dark. Furthermore, PLS regression analyses were conducted in order to study the possibility of modelling fingermark aging for potential fingermark dating applications. The resulting models showed an overall precision of \pm 3 days and clearly demonstrated their capability to differentiate older fingermarks (20 and 34-days old) from newer ones (1, 3, 7 and 9-days old) regardless of the substrate and lighting conditions. These results are promising from a fingermark dating perspective. Further research is required to fully validate such models and assess their robustness and limitations in uncontrolled casework conditions.
\end{abstract}

Keywords: fingerprint, kinetics, dating, Spearman correlation, PCA, PLSR

Corresponding author: aline.girod@unil.ch 


\section{Introduction}

Fingermark composition has been studied for decades because of its significance in the development of detection and enhancement techniques. This type of information is also required for research into fingermark aging and dating. Numerous analytical techniques have been used in order to gain more fundamental knowledge about initial fingermark composition. Gas chromatography mass spectrometry (GC/MS) [1-11], advanced mass spectrometry techniques (e.g. laser desorption, electrospray or atmospheric pressure chemical ionisation) [12-19], Raman spectroscopy [20-25] and Fourier Transform infrared spectroscopy combined with a microscope ( $\mu$-FTIR) [26-47] have been applied in this context. Other studies have also been carried out in order to study the changes occurring in fingermark composition over time. While GC/MS has often been used for this purpose [1-4, 7, 9, 48], the use of advanced mass spectrometry techniques $[12,14]$, liquid chromatography coupled with mass spectrometry (LC/MS) [49] and $\mu$-FTIR [36, 41, 43, 45, 46] have also been successful. Further details about these studies are summarized in a number of reviews [50-52].

All the above-mentioned techniques are useful in order to gain knowledge about fingermark composition. However, some of them are time-consuming, expensive and destructive. In fact, most chromatographic techniques (in particular GC/MS and LC/MS) require long sample preparation times and do not allow sample conservation after analysis, as fingermarks have to be extracted from their substrates using solvents. In contrast, spectroscopic techniques are faster to implement, cheaper and non-destructive, allowing the analysis of the same fingermark in its initial state and over time. This can be an asset for research into fingermark aging and dating. While a few studies have reported the use of Raman spectroscopy or Raman chemical imaging to analyse fingermark composition and contaminants, $\mu$-FTIR is the most commonly reported spectroscopic technique for fingermark analysis. For fingermark samples, $\mu$-FTIR is generally used in reflection or attenuated total reflection (ATR) modes, coupled with chemical imaging or not. The analysis of fresh fingermarks left on porous and nonporous substrates has been carried out by numerous authors, and better spectral quality has been reported for fingermarks left on metal and glass [26, 27, 33-37, 45, 53-55]. Further analyses have revealed that the main spectral features found in the fingermarks of various donors are very similar to each other, and correspond to esters and fatty acids. However, the relative amounts secreted from these two classes of compounds varied significantly between donors $[45,55]$. Differences in fingermark composition between children and adults were also noted [37, 41, 43]; residue from children contained far more eccrine volatile compounds (e.g. carboxylic acid salts, amino acids and proteins) than lipid compounds. While one study proposed the development of a regression model allowing the estimation of the age of a person between 4 and 68 years old based on these composition differences [37], other research found no significant age trend for people aged from under 20 years old to more than 50 years old (no precise ages were mentioned) [45]. This study also noted no significant variation in the lipid composition as a function of gender. Numerous studies have also been conducted to determine the ability of FTIR to analyse and identify extrinsic fingermark compounds, such as explosive residue [38-40, 42, 47], drugs [32] and other substances [36, $47,57]$. In the area of fingermark aging, only a few studies have been conducted using $\mu-$ FTIR. One study noted an overall decrease in the signal intensity of fingermark spectra over a 9-month period, probably due to evaporation of the fingermark deposit [45]. While this study also reported the greatest loss of lipid material during the first 3 months following deposition, no relevant classification of fingermarks based on age could be obtained using principal component analysis (PCA). Another study compared fingermark aging under ambient and vacuum conditions and observed that exposure to vacuum caused a significant reduction in 
the lipid composition of fingermarks, with the loss of tetradecanoic and pentadecanoic acid particularly noted $[41,46]$. The effect of temperature on the aging of fingermark residue (from room temperature to $80^{\circ} \mathrm{C}$ ) was also studied, and a general decrease in the absorbance of the main bands of lipid components was noted with increasing temperature; this was postulated to be the result of degradation to lower molecular weight compounds with subsequent volatilization [36]. Two studies focused on children's fingermarks [41, 43]. They noted that children's marks were very stable over time because salts were the most abundant compounds in them. Chemometric analyses using hierarchical cluster analysis (HCA) revealed that children's and adults' fingermarks were distinguishable for up to four weeks after deposition, based on differences in sebum composition.

All of these studies demonstrated that $\mu$-FTIR has the ability to analyse the composition of fresh and aged fingermarks. Vibrational bands corresponding to eccrine and sebaceous compounds have been identified in fresh fingermarks, while some aging processes have been briefly described in previous studies. However, few studies have actually focused on fingermark aging using $\mu$-FTIR. As a result, it would be particularly interesting to further investigate the capacity of $\mu$-FTIR to gain more fundamental knowledge about fingermark aging processes. Furthermore, since $\mu$-FTIR is a quick and non-destructive analytical technique available to most forensic science laboratories, it could also be an asset in the research and development of fingermark dating methodologies, as its implementation in practical contexts could easily be done at relatively low costs.

The aim of the current work was to use $\mu$-FTIR to study fingermark composition from its initial stage (fresh fingermarks) and over time (up to one month old fingermarks). The effects of two different acquisition modes (reflection and ATR) on the composition and variability of fresh fingermarks were first evaluated. Further, the influence of two substrates (aluminium foils and glass slides) and two different storage conditions (in the light and in the dark) on fingermark aging was investigated. As $\mu$-FTIR data are highly multivariate (hundreds of variables in each spectrum), the technique lends itself to chemometric processing for various sample types [41, 57-60]. In addition to visual observation of spectra, principal component analysis (PCA), correlation calculations and partial least squares regression (PLSR) were used in the current work to thoroughly assess the dataset structure. PCA and correlation calculations were used in a first step to assess the variability of fresh and aged fingermarks, to evaluate their similarity and whether they could be grouped by age. Based on these results, PLSR was then conducted in order to study the possibility of modelling infrared spectral data for potential fingermark dating applications.

\section{$\underline{\text { Materials and Methods }}$}

\section{Sampling: donor, data sets and deposition protocol}

This research was conducted according to a previously published formal framework for fingermark dating [61, 62]. This framework considers it impossible to build one aging model that works for all donors' fingermarks because of the large inter-variability of fingermarks from different donors. This framework rather suggests the building of a new aging model for each person. Therefore, in this study, the fingermarks of one single donor were used to study their initial composition and aging. This donor was a Caucasian female aged 27, with a typical diet and wearing no cosmetics. Fingermarks were first deposited on aluminium foil (as available in Australian supermarkets) because of the perfect reflective properties of this surface; these were left to age for $0,1,3,7,9,20$ and 34 days. Fingermarks were also 
deposited on glass (Livingstone microscope slide glass Pathology Grade) and left to age for 0, 1, 3, 7 and 9 days. This enabled a comparison of the effects of using different substrates. Two different acquisition modes were tested (reflection and ATR) and fingermarks were stored in two different conditions (in the dark and in the light). For each age, four fingermark specimens were deposited two by two on two different days and six spectra replicates were acquired per specimen, for a total of 24 spectra per age. No more than four fingermarks were deposited per day. Table 1 summarizes the acquired datasets:

\begin{tabular}{|c|c|c|c|c|c|c|c|c|}
\hline Dataset & Substrates & Mode & Storage & $\begin{array}{c}\text { Ages } \\
\text { [days] }\end{array}$ & $\begin{array}{c}\text { \# FM/age } \\
\text { (specimens) }\end{array}$ & $\begin{array}{c}\text { Deposition } \\
\text { days }\end{array}$ & $\begin{array}{c}\text { \# spectra/FM } \\
\text { (replicates) }\end{array}$ & $\begin{array}{l}\text { \# total } \\
\text { spectra }\end{array}$ \\
\hline 1 & Alu & Reflection & Light & $\begin{array}{l}0,1,3,7 \\
9,20,34\end{array}$ & 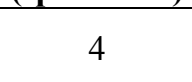 & $\begin{array}{l}\text { Day } 1 \\
\text { Day } 2\end{array}$ & & 168 \\
\hline 2 & Alu & Reflection & Dark & $\begin{array}{c}0,1,3,7 \\
9,20,34\end{array}$ & 4 & $\begin{array}{l}\text { Day } 1 \\
\text { Day } 2\end{array}$ & & 168 \\
\hline 3 & Alu & ATR & Light & $\begin{array}{l}0,1,3,7 \\
9,20,34\end{array}$ & 4 & $\begin{array}{l}\text { Day } 3 \\
\text { Day } 4\end{array}$ & & 168 \\
\hline 4 & Alu & ATR & Dark & $\begin{array}{l}0,1,3,7 \\
9,20,34\end{array}$ & 4 & $\begin{array}{r}\text { Day } 3 \\
\text { Day } 4\end{array}$ & 6 & 168 \\
\hline 5 & Glass & ATR & Light & $0,1,3,7,9$ & 4 & $\begin{array}{l}\text { Day } 5 \\
\text { Day } 6\end{array}$ & 6 & 120 \\
\hline 6 & Glass & ATR & Dark & $0,1,3,7,9$ & 4 & $\begin{array}{l}\text { Day } 5 \\
\text { Day } 6\end{array}$ & 6 & 120 \\
\hline
\end{tabular}

Table 1: Datasets acquired in this study

During fingermark deposition, the following standardized protocol was used:

1) The donor performed her tasks normally before deposition. The only condition was to avoid hand washing with soap within the last 45 min preceding the deposition.

2) Both thumbs were gently rubbed on the forehead and the edge of the nose, mimicking a natural movement.

3) The pressure and time of deposition were controlled. Each fingermark was deposited on a kitchen scale with an approximate pressure of $500 \pm 100 \mathrm{~g}$ for $15 \mathrm{~s}$.

4) The donor performed her tasks normally for another $30 \mathrm{~min}$.

5) A second deposition from each thumb was performed.

After deposition, fingermarks were directly analysed (15 to 30 minutes after deposition) and/or stored according to the chosen storage conditions.

\section{Fingermark analysis}

Analyses were carried out on a Thermo Scientific Nicolet Continuum infrared microscope equipped with a conventional liquid-nitrogen-cooled mercury cadmium telluride (MCT) detector. During ATR analysis, the tip of the germanium crystal touched the fingermarks in order to gather spectral information from the surface layer of the samples only.

128 scans were taken per sample with $4 \mathrm{~cm}^{-1}$ resolution over a range of $650-4000 \mathrm{~cm}^{-1}(1738$ variables). All the spectra were collected with the OMNIC 3.2 software and each was saved in *.SPA and *.CSV formats before being further processed.

Before all sample analyses, background spectra were acquired under the same conditions as the sample spectra from a clean (fingermark-free) area of the substrate. Furthermore, the sensitivity of the infrared microscope was monitored by analysing a controlled amount of cholesterol powder each time before each set of sample analyses. This allowed the establishment of a set of control intensities for specific vibrational bands (e.g., v(OH), 
$v(\mathrm{CH})_{\text {aliphatic }}$ and $\left.v(\mathrm{CH})_{\text {aromatic }}\right)$. This procedure ensured the quality and comparability of the acquired sample spectra.

\section{Processing steps}

Before any processing steps, all spectra were transformed from reflectance to absorbance units and presented as a function of wavenumber $\left(\mathrm{cm}^{-1}\right)$. Identification of the chemical compounds was then conducted, based on the frequency values found in the general FTIR literature [63-65] and in papers related to fingermark analysis [27, 36, 41, 43, 45]. Only the most intense bands were taken into consideration.

\section{Data pre-processing}

Before applying chemometric tools as PCA and PLSR, undesirable scattering effects (e.g. baseline shift or non-linearities), which strongly affect the sample spectra, were reduced by testing different pre-processing steps $[57,66]$. These included: normalisation, baseline correction (offset), $1^{\text {st }}$ and $2^{\text {nd }}$ derivative combined with Savitzky-Golay smoothing, Multiplicative Scatter Correction (MSC) and Standard Normal Variate (SNV). In this study, 18 different combinations of these techniques were eventually tested.

For each pre-processing combination and each dataset, the root mean square error (RMSE) and the coefficient of determination $\left(\mathrm{R}^{2}\right)$ for calibration and prediction were calculated using cross-validated partial least squares regression (PLSR) in order to choose the pre-processing protocol that gave the best-fitted model (i.e. the lowest RMSE and the highest $\mathrm{R}^{2}$ ) across the ages. These PLSR models were built on a subset of the data in order to accelerate the calculation processes. Replicate spectra from only one fingermark per age (i.e. six spectra per age) were used.

For each dataset, the best results were obtained using the Savitzky-Golay $2^{\text {nd }}$ derivative. This pre-processing corrects broad baseline features and allows overlapping peaks to be resolved because $2^{\text {nd }}$ derivative peaks are always narrower than the original spectral peaks $[40,66]$. The $2^{\text {nd }}$ Savitzky-Golay derivative was thus systematically applied on each spectrum before any other calculations.

\section{Data reduction - choice of variable intervals}

A single FTIR spectrum collected according to the parameters described above contains 1,738 variables. However, many of the variables are strongly correlated (represent the same chemical species) and many of them do not necessarily explain a significant part of the variance of the phenomenon under observation (i.e., effect of substrates, acquisition modes, ages and storage conditions). This is why variable selection has to be conducted in order to obtain better results, particularly when using chemometric tools. This study thus concentrated on manual data reduction, i.e. elimination of the spectral regions containing little or no chemical information, based principally on the spectra acquired from fresh fingermarks.

\section{Chemometrics}

After applying the pre-processing and data reduction steps, different statistical tools were applied using The Unscrambler ${ }^{\circledR}$ X (version 10.3, Camo ASA, Oslo, Norway) and R@ 3.03 for Macintosh software packages.

PCA is a commonly used unsupervised exploratory chemometric technique. The main utility of PCA is to reduce the dimensionality of multivariate datasets through the calculation of new 
latent variables, which are linear combinations of the original variables. These linear combinations, called principal components (PC), are calculated along the directions of maximum variance and are perpendicular to each other. PCA can be applied on highly correlated data, such as spectroscopic data [57], and their normality is not a strict requirement, as it is not a p-value driven technique [57, 67]. Thus, PCA allows the exploration of the data structure in order to detect sample groups and study their distribution along the different PCs (scores plots). Furthermore, by studying the coefficients of the linear combinations associated with each PC (i.e. loadings plots), the variables influencing the observed separations can be identified.

PCA was applied on fresh fingermarks to study the impact of the acquisition mode on the variability of the acquired spectra. The mode allowing the acquisition of the most reproducible spectra was chosen. Then, the effect of the substrate on the initial composition was assessed; i.e. whether fresh fingermark spectra could be grouped by substrate or not. Furthermore, PCA was also applied on aged fingermark spectra in order to explore potential groupings (by age) of fingermarks left on different substrates and stored under different lighting conditions. Variables influencing the separations were also identified and validation processes were conducted by internal cross-validation [68].

Correlation coefficients allow the measurement of the association between univariate or multivariate samples and are thus easily adapted to spectroscopic data. The correlation between the spectra of aged fingermarks acquired on different substrates and stored under different conditions was measured. In order to calculate the most appropriate coefficients, the normality of the datasets was tested using two different multivariate normality tests (Mardia and Royston test $[69,70])$. It was observed that the six datasets taken separately and as a whole did not follow the normality assumption. This result may be due to the fact that the aging kinetics of biological materials (e.g., lipids, solvents) follow exponential laws [71-74], which are a monotonic but non-linear functions and as such, do not correspond to a normal distribution. The Spearman rank correlation was therefore chosen because it is a nonparametric statistic measuring monotonic association between variables acquired at the ordinal level, and is thus applicable to non-normal datasets $[75,76]$. For better visualisation, the results are presented here as colour correlation matrices.

PLSR is a popular supervised chemometric technique well-adapted to multivariate and highly correlated datasets [77]. While similar to PCA, the main difference is that the dependent variables $\mathrm{Y}$ (in this study, the ages) are known. PLSR models simultaneously both the X- and $\mathrm{Y}$-matrices to find new $\mathrm{X}$-variables (independent or predictive variables) that will best predict the Y-variables, using linear transformations. These new latent variables are generally called PLSR components. PLSR actually maximizes the covariance between $\mathrm{X}$ and $\mathrm{Y}$ by mixing two PCA computations, one for X and one for Y [68]. PLSR with internal cross-validation [68] was carried out on aged fingermark spectra in order to study the possibility of modelling fingermark aging for potential fingermark dating applications.

\section{$\underline{\text { Results and discussion }}$}

\section{Initial composition of fingermarks}

In this study, the main vibrational bands identified in all fresh fingermark spectra were comparable to those identified in earlier research on fingermark composition [35, 41, 45]. Eccrine secretions were observed through N-H stretches at 3298 and $1541 \mathrm{~cm}^{-1}, \mathrm{C}-\mathrm{N}$ stretches at 1541 and $1200-1250 \mathrm{~cm}^{-1}$ and $\mathrm{C}=\mathrm{O}$ stretches at $1649 \mathrm{~cm}^{-1}$. These vibrations mainly 
represent secondary amides (e.g., proteins). It is important to note that these eccrine vibrational bands were not always visible in fresh fingermarks. In fact, some spectra showed only sebaceous material, as reported earlier [27]. Sebaceous material was indicated by C-H bands $\left(\mathrm{C}-\mathrm{H}, \mathrm{C}-\mathrm{H}_{2}\right.$ or $\left.\mathrm{C}-\mathrm{H}_{3}\right)$ at 1955, 2919, 2850, 1465, 1411 and $1379 \mathrm{~cm}^{-1}$. These bands showed the presence of fatty acids and wax esters. Furthermore, $\mathrm{C}=\mathrm{O}$ stretches were observed at $1741 \mathrm{~cm}^{-1}, \mathrm{C}-\mathrm{C}-\mathrm{O}$ stretches at 1246 and $1115 \mathrm{~cm}^{-1}$ and O-C-C peaks at 1116 and $1051 \mathrm{~cm}^{-}$ 1 . These peaks represent saturated esters and thus confirm the presence of lipid compounds. Based on these results, it was possible to identify the most informative regions of the spectra as being 1000-1850 $\mathrm{cm}^{-1}$ and $2700-3600 \mathrm{~cm}^{-1}$ (Figure 1 and Table 2). Thus, it was decided to concentrate on these areas in further chemometric analyses, reducing the number of variables from 1738 (entire spectrum) to 910 (selected areas).

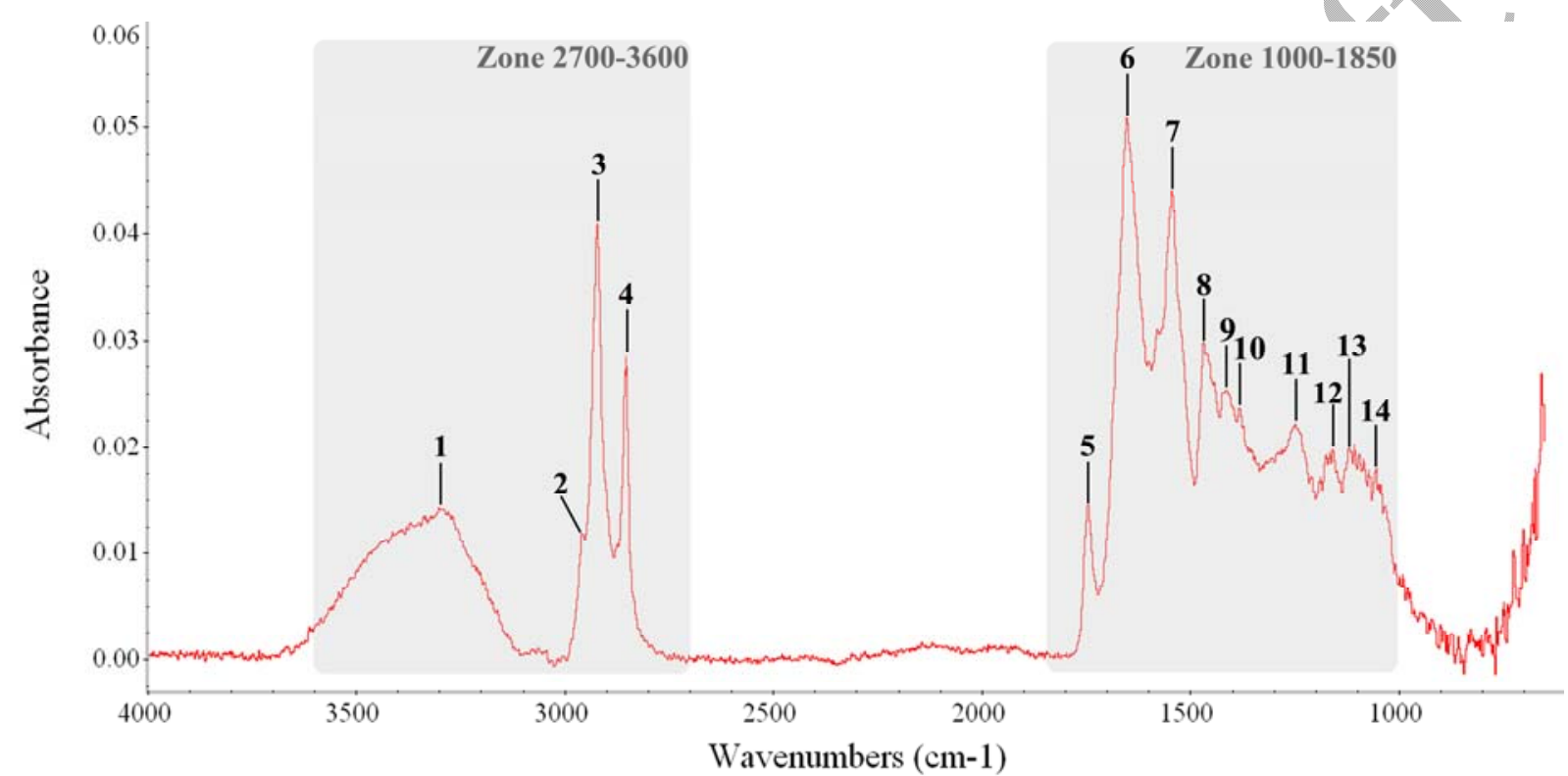

Figure 1: Example of a typical fresh fingermark spectrum containing sebaceous (peaks 2-5 and 8-11) and eccrine (peaks 1, 6 and 7) material. The main vibrational bands are numbered and identified in Table 1 . The shaded zones were selected for further chemometric analyses.

\begin{tabular}{|c|c|c|c|c|}
\hline Peaks & $\mathbf{c m}^{-1}$ & $\begin{array}{r}\text { Vibrations } \\
\end{array}$ & Compounds & Secretion \\
\hline 1 & $\begin{array}{l}3298 \\
3150-3600\end{array}$ & $\begin{array}{l}\text { Possible N-H stretch } \\
\text { Large water peak }\end{array}$ & Secondary amide & Eccrine \\
\hline 2 & 2955 & C- $\mathrm{H}_{2}$ stretch & Wax esters or fatty acids & Sebaceous \\
\hline 3 & 2919 & $\mathrm{C}-\mathrm{H}$ stretch $\left(1^{\mathrm{st}}\right.$ carbon $)$ & Long aliphatic $\mathrm{C}$ chains & Sebaceous \\
\hline 4 & 2850 & C-H stretch ( $2^{\text {nd }}$ carbon $)$ & Long aliphatic $\mathrm{C}$ chains & Sebaceous \\
\hline 5 & 1743 & $\mathrm{C}=\mathrm{O}$ stretch & Saturated esters & Sebaceous \\
\hline 6 & 1649 & $\mathrm{C}=\mathrm{O}$ stretch & Secondary amide & Eccrine \\
\hline 7 & 1541 & $\mathrm{~N}-\mathrm{H}$ and $\mathrm{C}-\mathrm{N}$ & Secondary amide & Eccrine \\
\hline 8 & 1465 & $\begin{array}{l}\mathrm{C}-\mathrm{H}_{3} \text { (asymmetric bend) } \\
\mathrm{C}-\mathrm{H}_{2} \text { (symmetric bend) }\end{array}$ & Aliphatic $\mathrm{C}$ chains & Sebaceous \\
\hline 9 & 1411 & $\mathrm{C}-\mathrm{H}_{3}$ (asymmetric. bend) & Aliphatic $\mathrm{C}$ chains & Sebaceous \\
\hline 10 & 1379 & $\mathrm{C}-\mathrm{H}_{3}$ (symmetric bend) & Aliphatic $\mathrm{C}$ chains & Sebaceous \\
\hline 11 & $\begin{array}{l}1246 \\
1200-1250\end{array}$ & $\begin{array}{l}\text { C-C-O stretch (asymmetric bend) } \\
\text { C-N stretch }\end{array}$ & $\begin{array}{l}\text { Esters } \\
\text { Secondary amide }\end{array}$ & $\begin{array}{l}\text { Sebaceous } \\
\text { Eccrine }\end{array}$ \\
\hline 12 & 1155 & C-C-O stretch & Saturated esters & Sebaceous \\
\hline 13 & 1116 & O-C-C stretch & Saturated esters & Sebaceous \\
\hline 14 & 1051 & $\mathrm{O}-\mathrm{C}-\mathrm{C}$ stretch & Saturated esters & Sebaceous \\
\hline
\end{tabular}

Table 2: Major vibrational bands corresponding to eccrine and sebaceous material observed in the IR spectra of fresh fingermarks. 
Similar sebaceous spectra were obtained using the two acquisition modes, but while spectra containing both eccrine and sebaceous compounds were obtained using ATR, no eccrine material could be observed using reflection (Figure 2A and 2B). Furthermore, as previously reported [78], ATR yielded better-resolved and more detailed spectra than reflection. Comparing the substrates (aluminium or glass), results were obtained using ATR and reflection on aluminium, but, as expected, only ATR yielded good quality spectra on glass, due to the highly absorbing nature of this substrate. It is also important to note that visual observations of the ATR spectra did not reveal any significant influence due to substrate (Figures 2B and 2C). The only observed difference was the large band located at around 1000 $\mathrm{cm}^{-1}$ on glass but not aluminium foil, which was due to Si-O stretching vibrations in glass. It was also noted that the presence of the large water peak visible at $3150-3600 \mathrm{~cm}^{-1}$ was not consistent, whatever the acquisition mode or substrate. This may be due to non-homogenous repartition of water on fingermark residue.
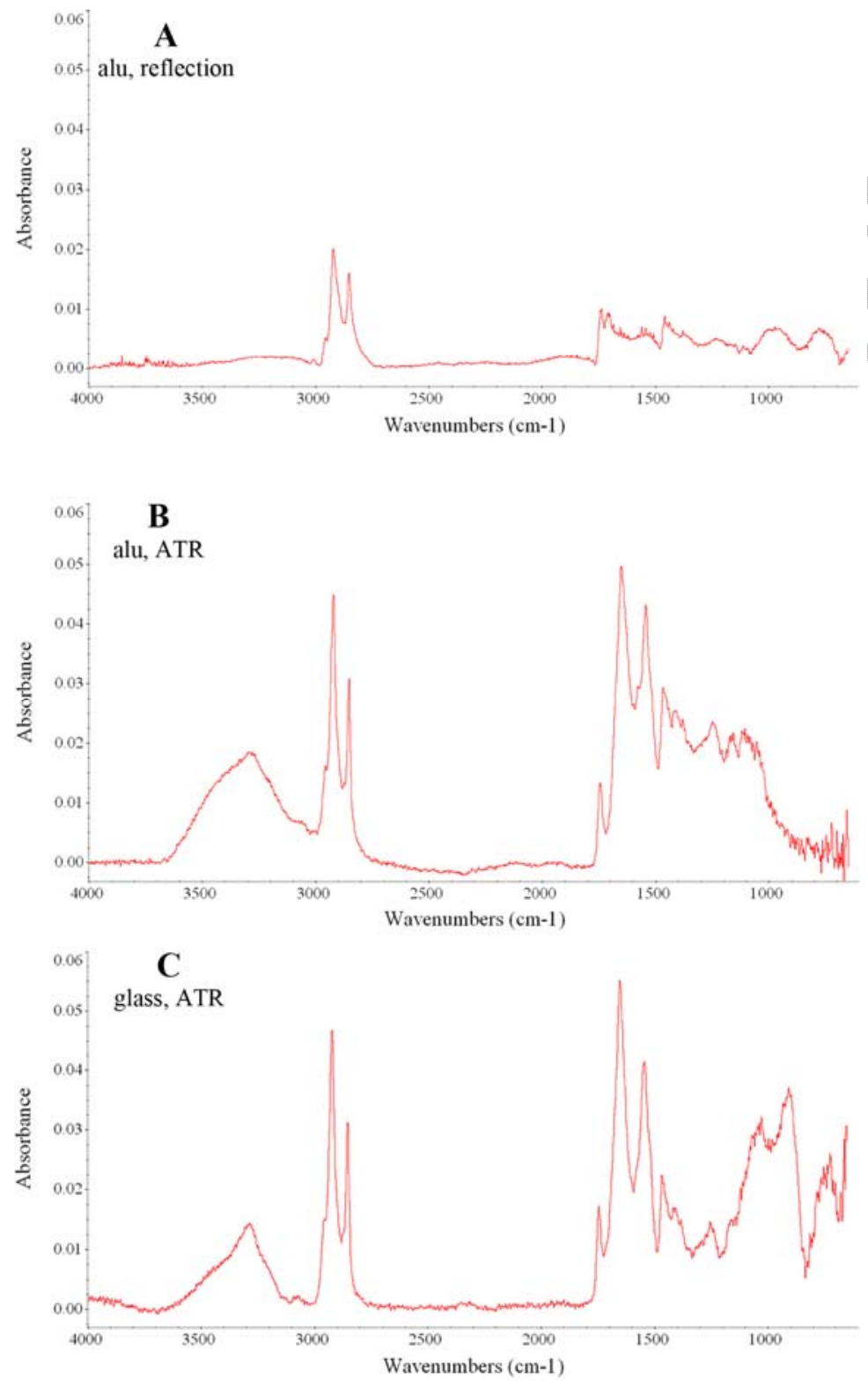

Figure 2: Examples of typical spectra obtained after analysis of fresh fingermarks on A) aluminium with reflection, B) aluminium with ATR and C) glass with ATR. 
The visible differences between reflection and ATR modes were corroborated by using PCA on all spectra of fresh fingermarks left on aluminium on two different days (acquisition day 1 and 2). In fact, ATR and reflection spectra were clearly separated when plotting PC1 and PC2, which together accounted for 39\% of the total variance (Figure 3). The ATR spectra were grouped along PC1, while the PC2 separated the reflection spectra based on acquisition day. This second separation can potentially be explained by differences from the donor over the two acquisition days (day 1 and day 2). This difference is however less visible on ATR spectra, which were also acquire on two different days (day 3 and day 4). This can mean that donor composition differences can appear between two different days, but are irregular. This observation is compatible with the large intra-variability of fingermark samples already reported in previous work $[4,9,11,41,45]$ and should be further studied in order to better understand its origin. By studying the loading plots, it was observed that the $\mathrm{C}-\mathrm{H}, \mathrm{C}-\mathrm{H}_{2}$ and $\mathrm{C}-\mathrm{H}_{3}$ bands (sebaceous compounds), as well as the $\mathrm{C}=\mathrm{O}$ (sebaceous and eccrine compounds) stretches had the most significant influence on the separation based on PC1 and PC2 (see Figure 1 in the supplementary data).

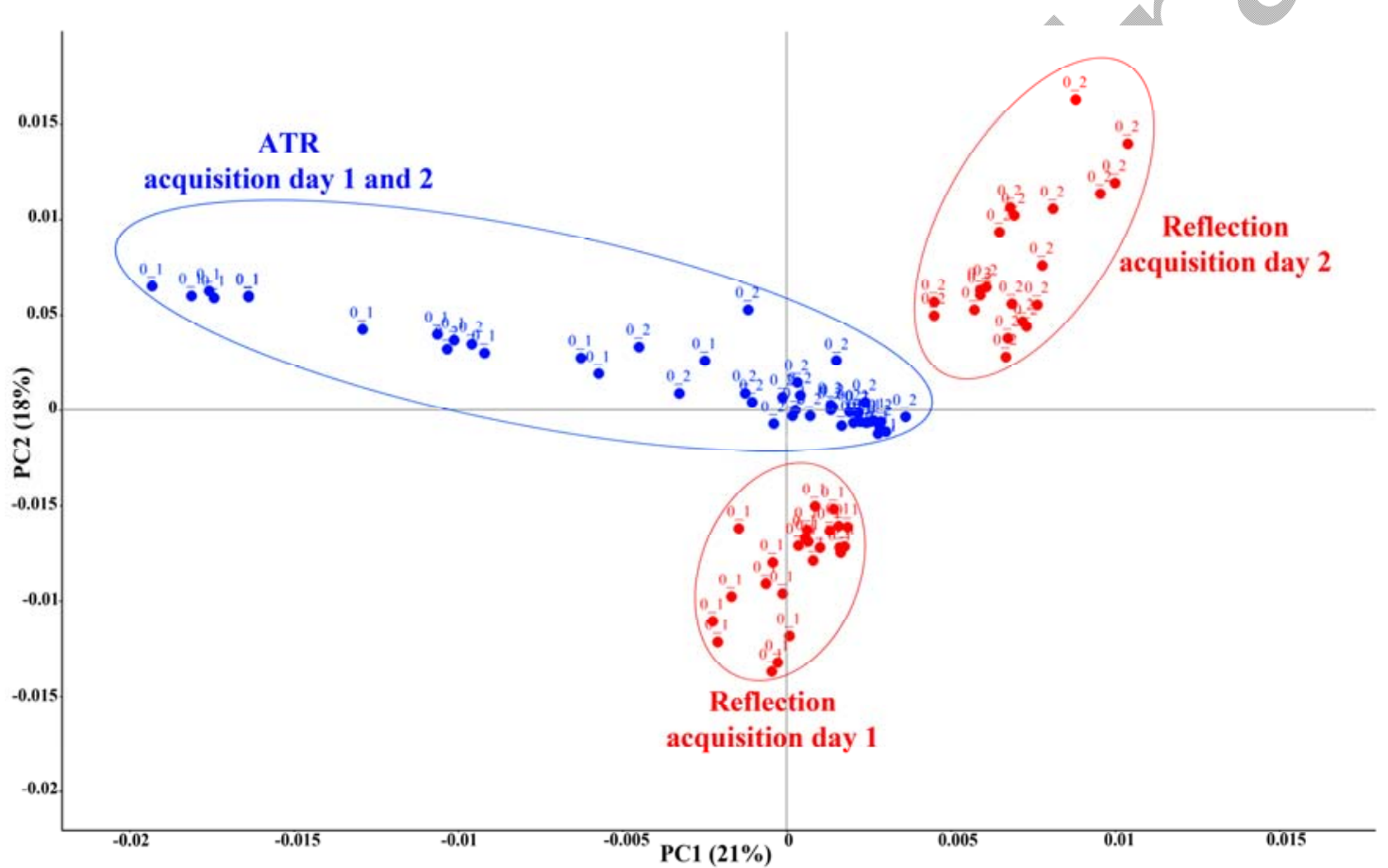

Figure 3: PCA scores plot of fresh fingermarks left on aluminium and acquired using FTIR in ATR and reflection mode on two different days. Three main groups were detected when plotting PC1 and PC2: all ATR spectra (independently from the acquisition day), reflection spectra acquired at day 1 and reflection spectra acquired at day 2 .

As only the ATR mode allowed the acquisition of spectra of fingermarks left on both glass and aluminium, and since it proved to be more sensitive than reflection, further analyses were carried out using only ATR spectra. As a result, the substrate effect on fresh fingermarks was assessed by conducting another PCA experiment on ATR spectra only. However, this analysis did not reveal any differentiation between samples left on aluminium or glass, as no groups could be separated. This indicated that the tested substrates did not significantly influence the acquired fresh fingermark spectra.

\section{Aged composition of fingermarks}

The spectra of aged fingermarks originally containing mainly lipid compounds (i.e., sebaceous secretions) showed a decrease in the intensity of all vibrational bands (Figure 4A), 
while spectra containing both eccrine and sebaceous compounds showed the same decrease in lipid peak intensity, and the complete disappearance of eccrine material over time (Figure 4B). Comparing the effects of substrates (aluminium and glass) and storage conditions (light and dark), no significant visual differences were observed over time. However, chemometric processing was carried out in order to further study these datasets and the results are presented below.

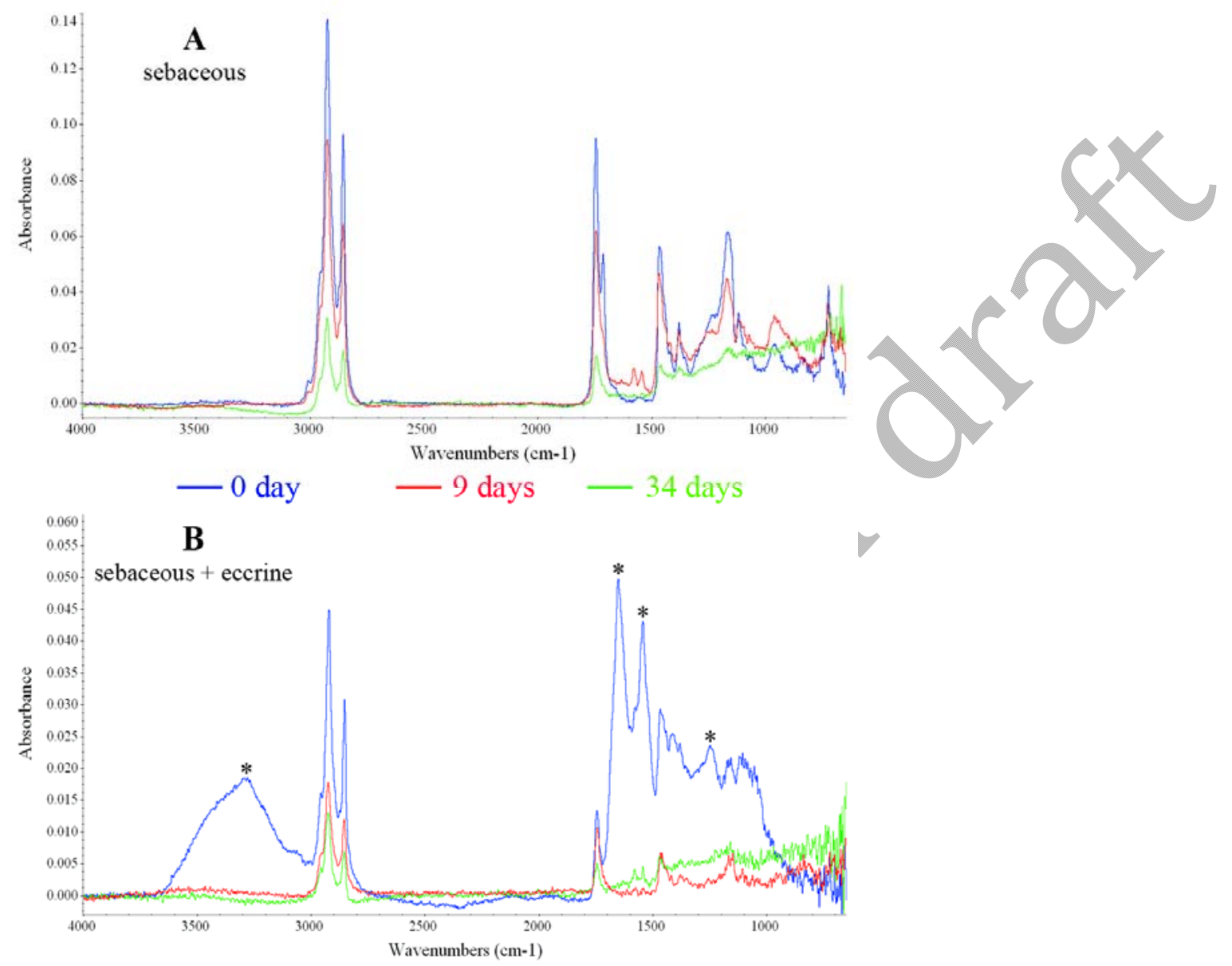

Figure 4: Examples of the aging pattern for fingermark spectra containing mainly A) sebaceous material and B) sebaceous and eccrine $\left(^{*}\right)$ material.

\section{Correlations between fingermarks according to their age}

Spearman correlation coefficients were calculated and colour matrices were computed to study the effects of substrates and storage conditions on aging. The average spectra of the six replicates acquired per fingermark were used in order to obtain four results per age and allow better visualisation of sample correlations.

Correlation matrices were calculated for fingermark samples aged from 1 to 9 days on aluminium and glass, and stored in the light (Figure 5A) and in the dark (Figure 5B). The highest correlation coefficients, between 0.6 and 1 (marked in medium to dark blue in Figure 5 ), were found between replicates of the same age, and not between replicates of different ages (i.e., those coefficients were under 0.6). This indicated that fingermarks were correlated by age regardless of the substrate and storage conditions. It was interesting to note that for storage in the light, the correlation coefficients between fingermarks of different ages were very low on glass (i.e., between 0 and 0.2 , marked in green colour) and slightly higher on aluminium (i.e., up to 0.4, marked in light blue) (Figure 5A). In other words, the 
differentiation between ages was stronger on glass than on aluminium. Furthermore, high correlation coefficients were also obtained between samples left on different substrates in the light (i.e., fingermarks on aluminium compared to fingermarks on glass; see the pattern in the "alu-glass" section of the matrix in Figure 5A). This indicates similar aging patterns for the substrates. On the contrary, for fingermarks stored in the dark, almost none of the correlation coefficients between samples left on different substrates were high (the "alu glass" section in Figure 5B). These results indicate that the aging of fingermarks was dependent on the substrate when stored in the dark but not when stored in the light. One possible explanation might be that aging caused larger differences in fingermarks stored in the light, thus hiding the smaller substrate effects.

Further observations showed that fingermark aging was significantly influenced by storage conditions. In fact, the correlation coefficients obtained for fingermarks left on aluminium from 1 to 34 days were higher between samples having the same age and storage conditions (see medium to dark blue colours in the matrix in Figure 6). On the other hand, no high coefficients were computed between samples stored in the light and in the dark (see light-dark in the matrix in Figure 6). The same clear tendencies were observed for fingermarks left on glass, i.e. higher correlation coefficients between samples having the same age and being stored under the same conditions (see Figure 5 in supplementary data). These observations meant that fingermark spectra were changing over time and that these changes were dependent on the storage conditions.

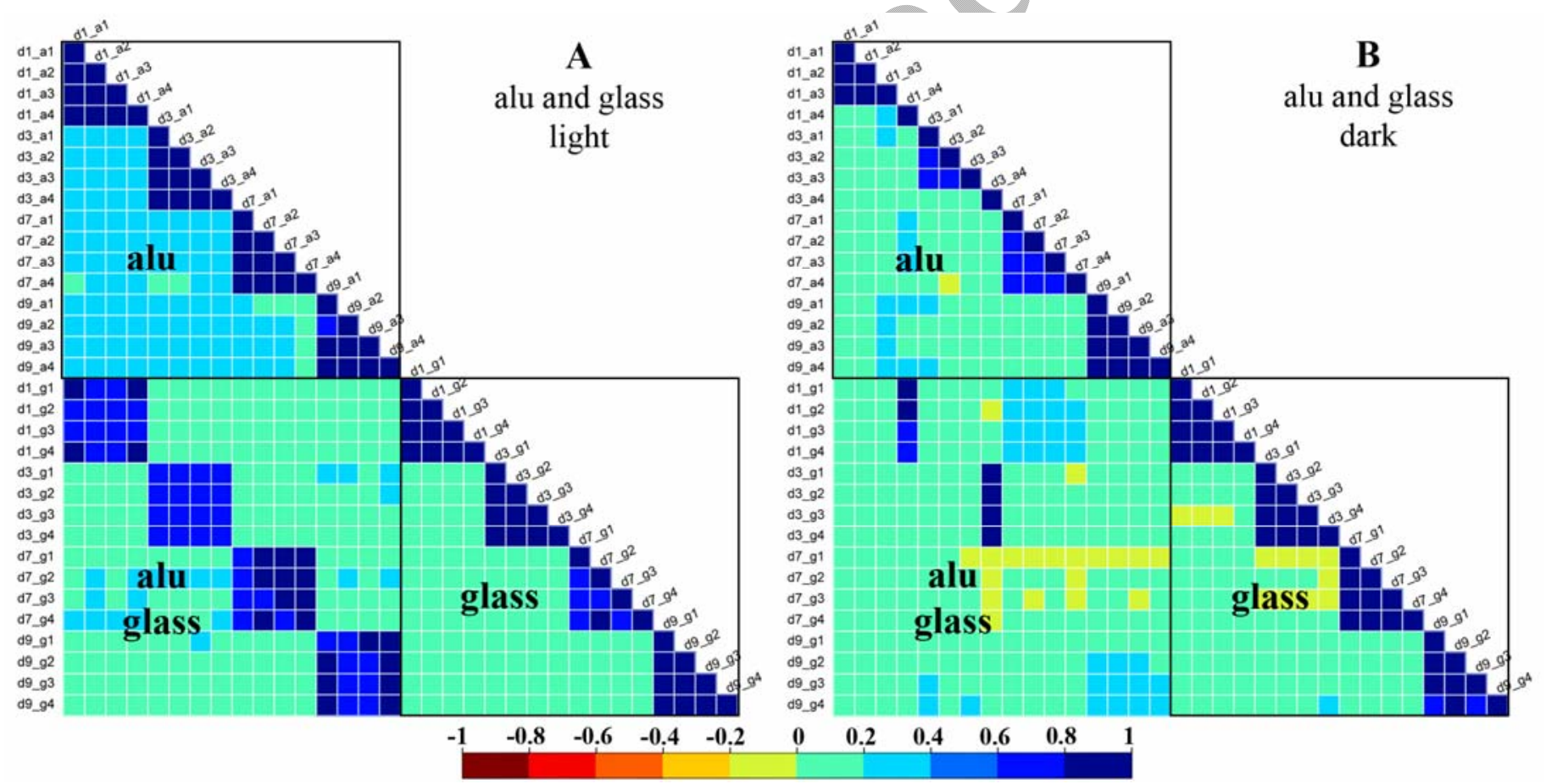

Figure 5: Spearman correlation matrices for the spectra of fingermarks deposited on aluminium $(a)$ or glass $(g)$ and acquired with ATR after 1, 3, 7 and 9 days $(d)$ of storage $\mathbf{A}$ ) in the light or B) in the dark. High correlation coefficients were generally obtained between specimens of the same age on the same substrate, and also between specimens on different substrates when stored in the light (value above 0.6). Correlations were lower between specimens of different ages (values below 0.6), and generally also between specimens left on different substrates when stored in the dark. 


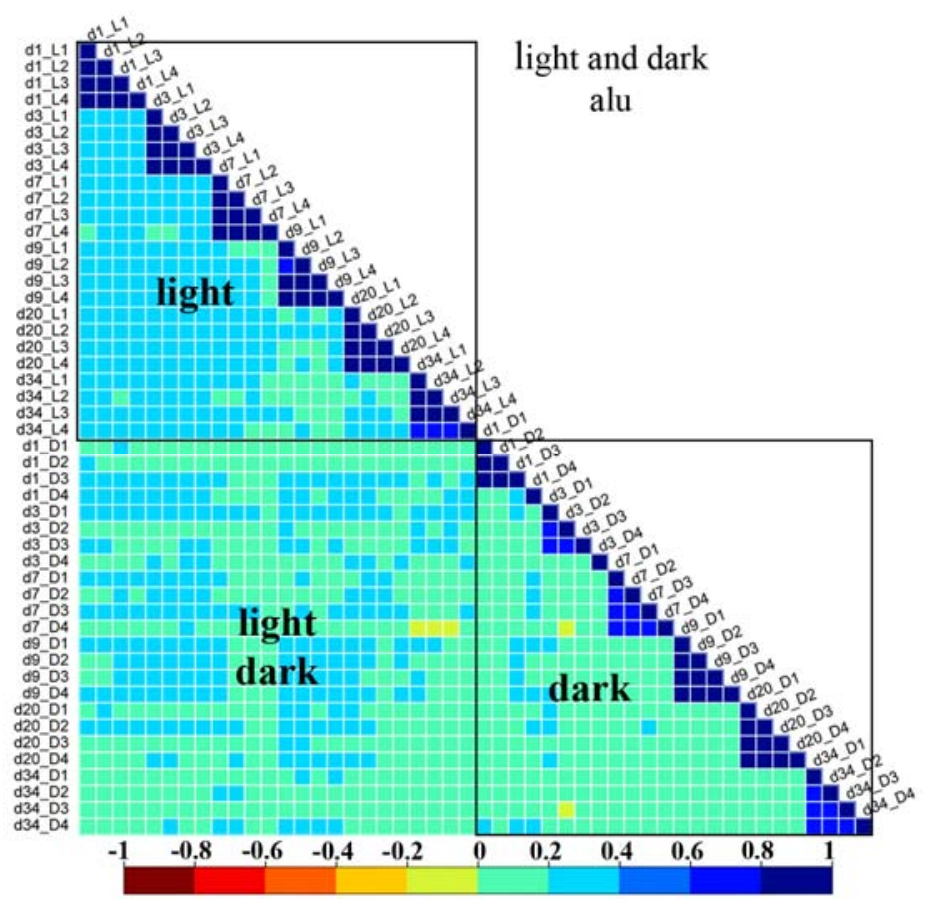

Figure 6: Spearman correlation matrices for the spectra of fingermarks deposited on aluminium and acquired with ATR after 1, 3, 7, 9, 20 and 34 days $(d)$ of storage in the light $(L)$ or in the dark $(D)$. High correlation coefficients were only obtained between specimens of the same age stored under the same storage conditions (values over 0.6).

\section{Classification of fingermarks according to their age}

PCA was conducted in order to further explore fingermark aging and the effects of substrate and storage conditions. The spectra from all fingermark specimens from 1 to 34 days old on aluminium and 1 to 9 days old on glass were used.

The PCA scores plot for fingermark specimens stored in the light exhibited a clear age separation using PCs 2, 4 and 5 (Figure 7). Aluminium and glass specimens were grouped together, showing again that substrate had no influence on aging for this storage condition. PCs 2, 4 and 5 accounted together for $20 \%$ of the total variance, meaning that age differences were responsible for only $20 \%$ of the total variance available in the dataset. Age was thus not the main source of variability, but still brought enough differences to allow age grouping. The other $80 \%$ of the variance could have many origins, such as the large intra-variability of fingermark specimens due to different factors, as previously reported in other studies $[4,9$, $11,41,45]$. 

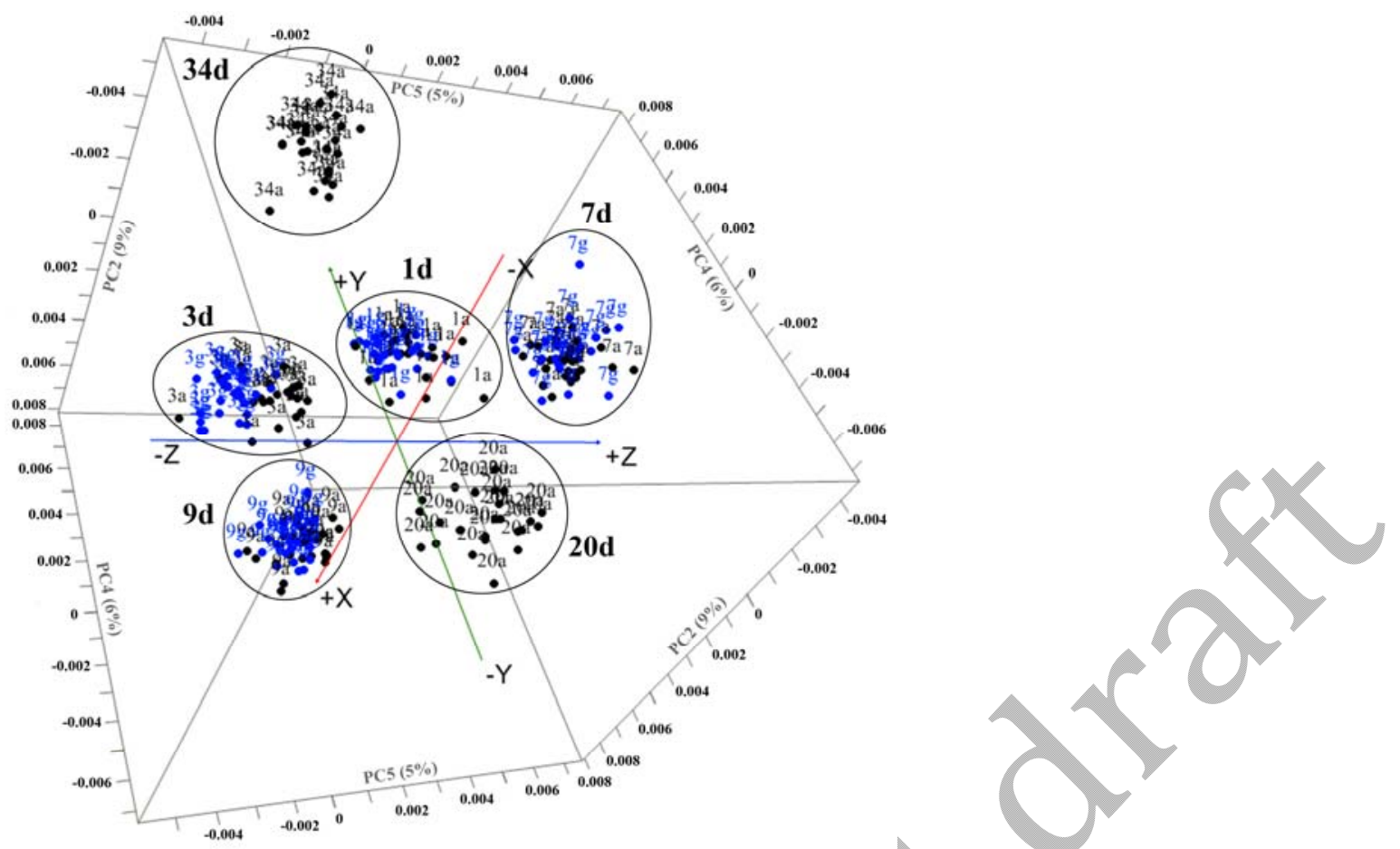

Figure 7 - Aging in the light from 1 to 34 days: PCA score plot of FTIR spectra (acquired with ATR) of fingermark specimens stored in the light over 9 days on glass (g, blue) and 34 days on aluminium (a, black). PCs 2, 4 and 5 allowed the separation of all ages into 6 groups $(1,3,7,9,20$ and 34 days). No effect of substrate was observed; specimens of the same age were grouped together no matter which substrate they were deposited on.

Results obtained from fingermarks stored in the dark were again more ambiguous than those from fingermarks stored in the light. In fact, while the fingermarks left on glass could be grouped by age using PCs 1-3, no PC combination allowed the age-based separation of aluminium specimens (Figure 8A). In fact, nearly all of the aluminium specimens stayed together in one main group in the middle of the scores plot. Only 1-day and some 7-days old fingermarks were separated from this main group using PCs 1-3, and were actually grouped together with 1-day old specimens on glass. These observations highlighted again the effects of substrate on the aging of fingermarks in the dark. However, in the PC 4-6 scores plot (Figure 8B), a clear separation of the older specimens (20 and 34-days old fingermarks) deposited on aluminium was possible, while younger specimens left on aluminium and glass were grouped together in the middle of the plot. PCs 1-3 accounted together for $24 \%$ of the total variance and PCs 4-6 for 14\%. Age differences and substrate effects were thus responsible for $38 \%$ of the variability of this dataset. The $62 \%$ of variance left may again be explained by the large variability of fingermark specimens as explained above.

These observations indicated that fingermarks could be grouped by age when stored in the light, regardless of the substrate, while specimens stored in the dark showed a substrate dependency. This corresponds to what was found using Spearman correlation coefficients. However, Spearman coefficients allowed the age separation of younger samples on aluminium. The fact that this was not possible with PCA might be due to the high influence of 20 and 34-days old fingermarks on the separation. Therefore, another PCA was conducted without these older specimens (Figure 9). In this new PCA, a good separation of younger aluminium samples was visible in the scores plot using PCs 4-6, while glass samples mostly remained unseparated in the middle of the plot, except for 1-day old specimens. PCs 4-6 accounted for $15 \%$ of the variability and represented the variability due to aging effects of younger aluminium samples. 
In summary, the PCA results showed that fingermarks could be grouped by age, but that aging was significantly influenced by storage conditions and also by substrate when specimens were stored in the dark.
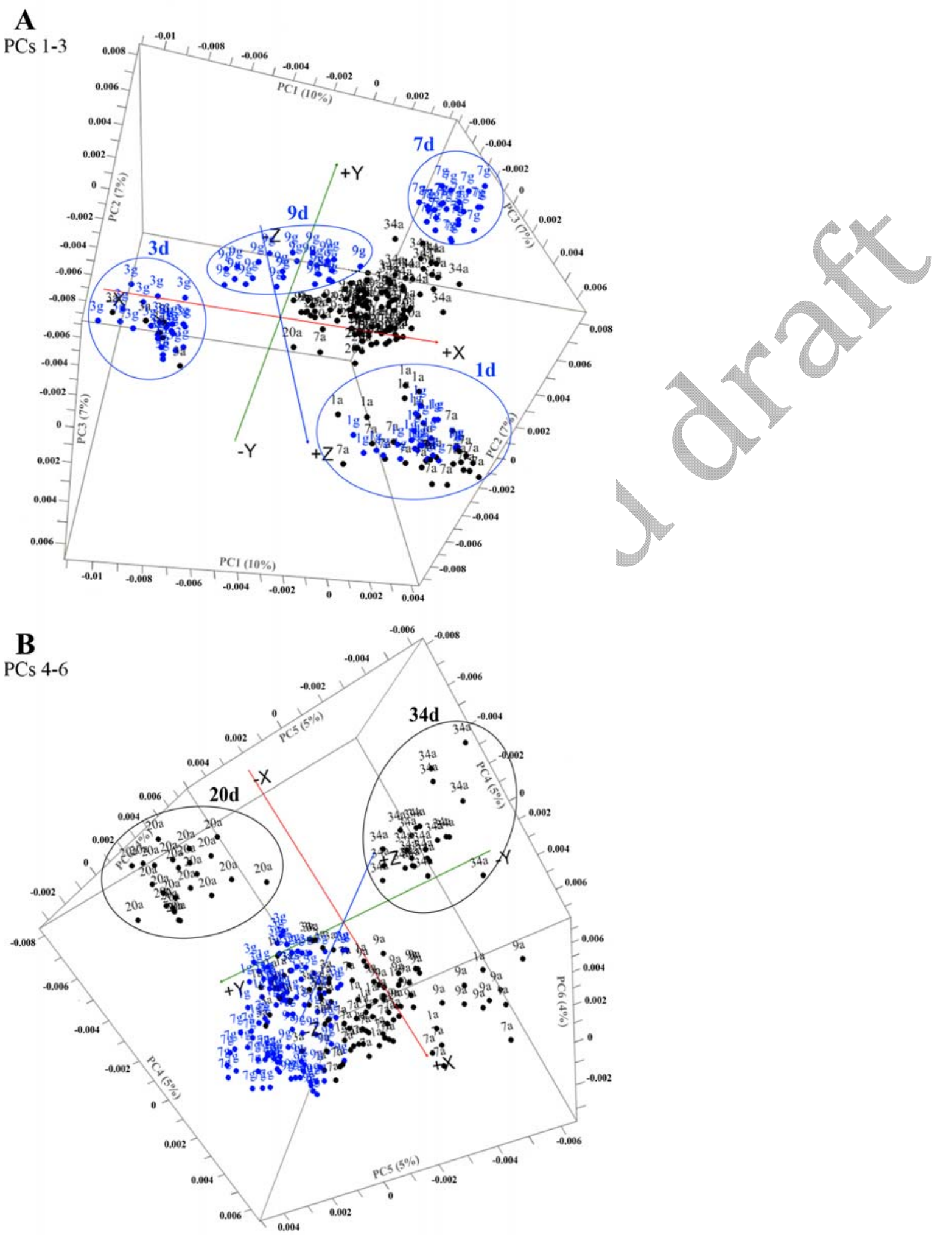

Figure 8 - Aging in the dark from 1 to 34 days: PCA scores plot for FTIR spectra (acquired with ATR) of fingermark specimens stored in the dark for 9 days on glass ( $\mathrm{g}$, blue) and 34 days on aluminium (a, black).

(A) PCs 1-3 allowed the age separation of glass samples.

(B) PCs 4-6 allowed the separation of 20d and 34d aluminium samples. 

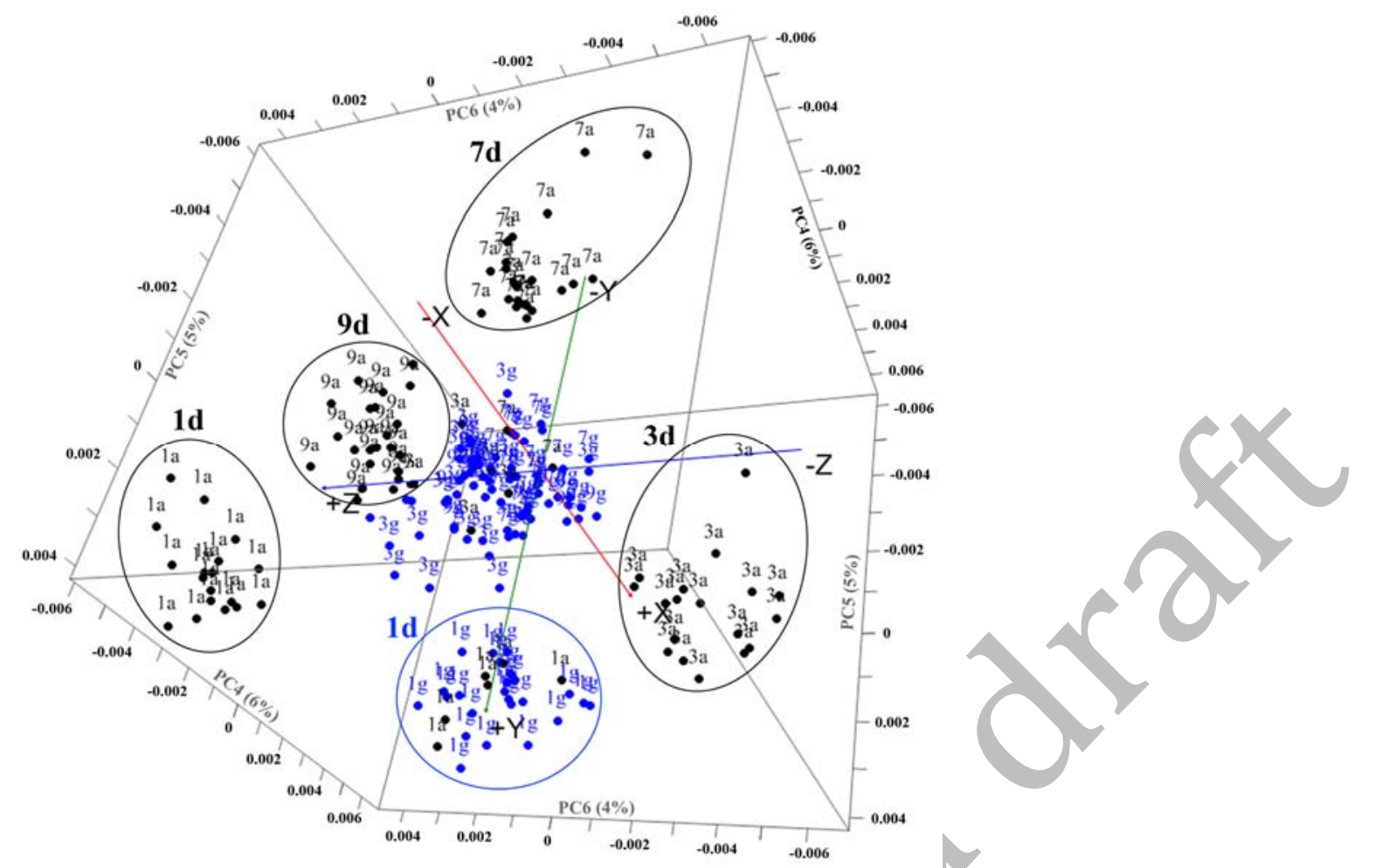

Figure 9 - Aging in the dark from 1 to 9 days: PCA scores plot of FTIR spectra (acquired with ATR) of fingermark specimen stored in the dark for 9 days on glass (g, blue) and aluminium (a, black). PCs 4-6 allowed the separation of aluminium samples by age (1,3, 7 and 9 days old), and of 1-day old glass specimens.

In order to further understand the observations made in Figures 7-9, the loadings plots of the relevant PCs (see Figures 2-4 in the supplementary data) were studied in order to identify the variables influencing the separations. Five groups of influencing variables were identified from the FTIR spectra:

(1) $\mathrm{C}-\mathrm{H}$ and $\mathrm{C}-\mathrm{H}_{2}$, sebaceous,

(2) $\mathrm{C}=\mathrm{O}$, sebaceous and eccrine,

(3) N-H and C-N, eccrine,

(4) $\mathrm{C}-\mathrm{H}_{3}$, sebaceous,

(5) C-C-O and O-C-C sebaceous.

The impact of these variables on aging is summarized in Table 3.

\begin{tabular}{|c|c|c|c|c|}
\hline & \multicolumn{2}{|c|}{ Aging in the light } & \multicolumn{2}{|c|}{ Aging in the dark } \\
\hline & Aluminium & Glass & Aluminium & Glass \\
\hline $\begin{array}{c}\text { Mains } \\
\text { variables }\end{array}$ & $\begin{array}{l}\mathrm{C}=\mathrm{O} \\
\mathrm{N}-\mathrm{H} \text { and } \mathrm{C}-\mathrm{N} \\
\mathrm{C}-\mathrm{C}-\mathrm{O} \text { and } \mathrm{O}-\mathrm{C}-\mathrm{C}\end{array}$ & $\begin{array}{l}\mathrm{C}=\mathrm{O} \\
\mathrm{N}-\mathrm{H} \text { and } \mathrm{C}-\mathrm{N} \\
\mathrm{C}-\mathrm{C}-\mathrm{O} \text { and } \mathrm{O}-\mathrm{C}-\mathrm{C}\end{array}$ & $\begin{array}{l}\mathrm{C}-\mathrm{H} \text { and } \mathrm{C}-\mathrm{H}_{2} \\
\mathrm{C}=\mathrm{O} \\
\mathrm{N}-\mathrm{H} \text { and } \mathrm{C}-\mathrm{N} \\
\mathrm{C}-\mathrm{H}_{3} \\
\mathrm{C}-\mathrm{C}-\mathrm{O} \text { and } \mathrm{O}-\mathrm{C}-\mathrm{C}\end{array}$ & $\begin{array}{l}\mathrm{C}-\mathrm{H} \text { and } \mathrm{C}-\mathrm{H}_{2} \\
\mathrm{C}=\mathrm{O} \\
\mathrm{N}-\mathrm{H} \text { and } \mathrm{C}-\mathrm{N} \\
\mathrm{C}-\mathrm{C}-\mathrm{O} \text { and } \mathrm{O}-\mathrm{C}-\mathrm{C}\end{array}$ \\
\hline
\end{tabular}

Table 3: Summary of the vibrational bands (variables) influencing aging of fingermarks deposited on aluminium and glass and stored in the light and dark.

$\mathrm{C}=\mathrm{O}, \mathrm{N}-\mathrm{H}$ and $\mathrm{C}-\mathrm{N}$ and $\mathrm{C}-\mathrm{C}-\mathrm{O}$ and $\mathrm{O}-\mathrm{C}-\mathrm{C}$ stretches were responsible for the age separation on both substrates in the light and in the dark. These bonds mainly correspond to saturated esters from sebaceous compounds and secondary amides from eccrine compounds. The $\mathrm{C}-\mathrm{H}$ 
and $\mathrm{C}-\mathrm{H}_{2}$ stretches correspond to long aliphatic carbon chains from sebaceous compounds and also had an impact on the separation according to age, but only when the fingermarks were stored in the dark. The $\mathrm{C}-\mathrm{H}_{3}$ stretches corresponding mainly to lipids also influenced the age separation of fingermarks stored in the dark, but only for specimens left on aluminium. Thus, these observations indicated that the separation of fingermarks according to their age was based on both sebaceous and eccrine compounds, while differences due to storage conditions and substrates were mainly caused by sebaceous compounds. Furthermore, the loadings also highlighted differences concerning the impact of the storage conditions (light or dark). In fact, $\mathrm{C}-\mathrm{H}$ bonds $\left(\mathrm{C}-\mathrm{H}, \mathrm{C}-\mathrm{H}_{2}\right.$ and $\mathrm{C}-\mathrm{H}_{3}$, sebaceous compounds) influenced the separation in the dark but apparently not in the light. This observation might indicate that $\mathrm{C}-\mathrm{H}$ differences only caused small variability in the spectra and were thus overwhelmed by larger impacts from other variables when the fingermarks were stored in the light. In fact, esters $(C=O)$ and eccrine compounds $(\mathrm{N}-\mathrm{H}, \mathrm{C}-\mathrm{N})$ quickly degrade in the light, causing the relevant peaks to disappear, with a large impact on the spectra. This could possibly mask the C-H variability. On the other hand, when fingermarks were aged in the dark, esters and eccrine compounds caused less drastic differences and the impact of $\mathrm{C}-\mathrm{H}$ degradation remained important for the separation.

\section{Modelling of fingermark aging}

Since Spearman coefficients and PCA indicated that fingermarks were correlated and could be grouped by age, aging modelling was trialled using PLSR. These models might represent a first step in the development of a fingermark dating methodology [61, 62] and should be as robust as possible to variations in storage conditions, as these impact on the aging of fingermarks but cannot be strictly controlled. On the contrary, the impact of the substrate is less important, as it might precisely be known in a particular case context and could thus be taken into account when building the model. Thus, independent PLSR models were built for each substrate. First, models were developed using fingermarks deposited on aluminium for 1 , 3, 7, 9, 20 and 34 days and stored in the light (Figure 10A), in the dark (Figure 10B) and in both storage conditions (Figure 10C). 

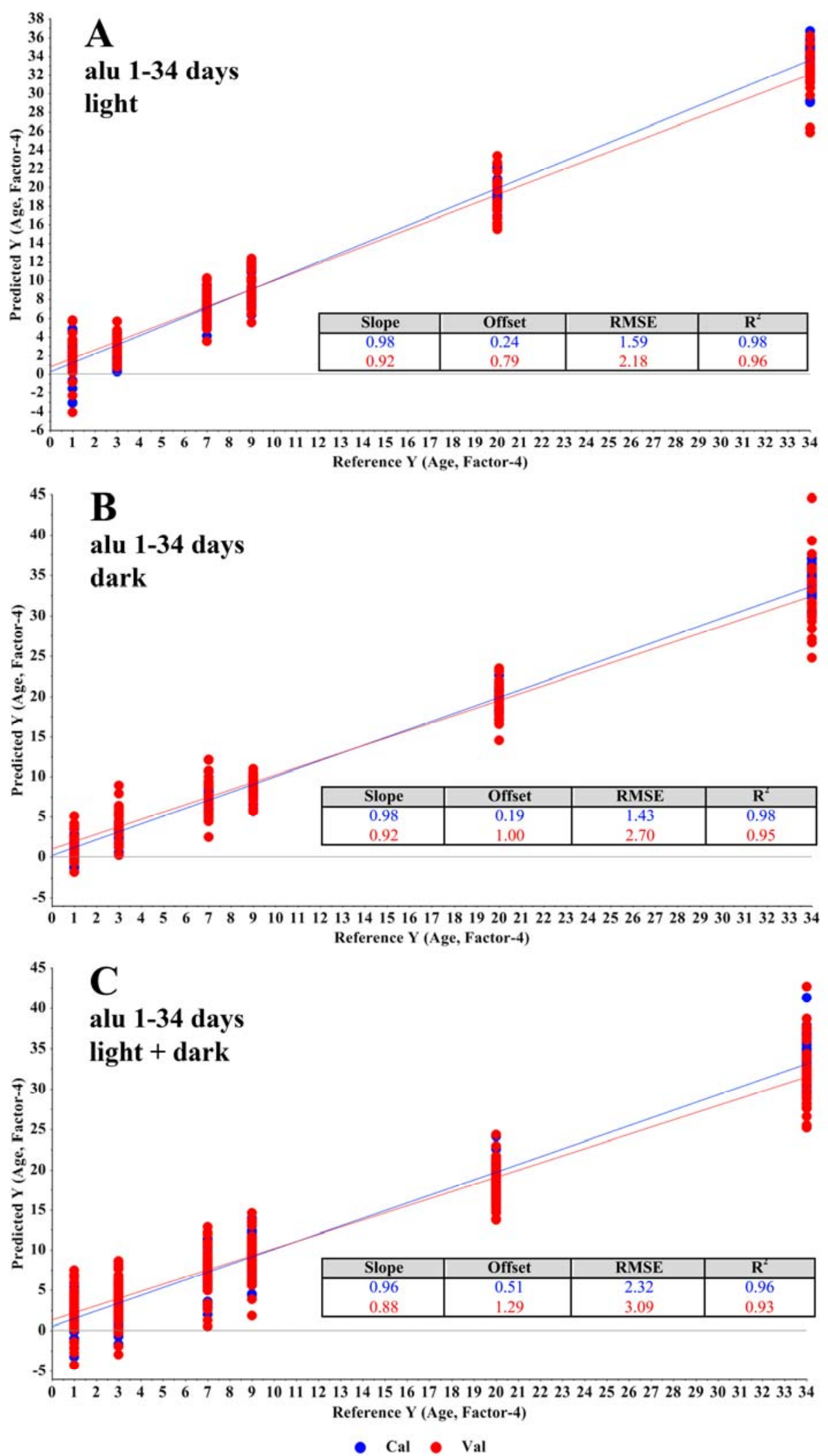

Figure 10 - Aging on aluminium from 1 to 34 days: PLS regression models obtained using fingermark spectra from 1 to 34 days old deposited on aluminium, acquired with ATR and stored A) in the light, B) in the dark and C) in the light or in the dark. Calibration (Cal, blue) and cross-validation (Val, red) points are shown. RMSE: root mean square error, $\mathrm{R}^{2}$ : coefficient of determination. 
Good linearity was obtained for the different storage conditions, as the $\mathrm{R}^{2}$ values were all above 0.90 (Figure 10). The Root Mean Square Error of validation (RMSEV) expresses the general model error and was \pm 2.18 days in the light, \pm 2.70 days in the dark and \pm 3.09 days for the combination of the two storage conditions ${ }^{1}$. Thus, the model precision decreased when combining storage conditions, because the variability of each predicted age increased. In the light, 20 and 34-day old fingermarks could be completely separated from 1, 3, 7 and 9-days old specimens but overlapped with each other (Figure 10A). Values obtained for fingermarks 9-days old and younger also showed overlap and the individual ages were thus not clearly differentiable either. In the dark (Figure 10B) and when combining both storage conditions (Figure 10C), 34-day old fingermarks could not be separated from the 20-day old ones but were differentiable from 9, 73 and 1-day old specimens, while 20-day old fingermarks could be separated from 0, 1 and 3-day old fingermarks, but not from 7 and 9-day old specimens.

It is important to note that an extreme Y-value (in our case, age) can influence PLS regressions. In this study, 20 and 34-day old fingermarks yielded significantly larger values than younger fingermarks. Thus, new PLSR models were built without these specimens (Figure 11). The main parameters of these new PLSR models were comparable to those represented in Figure 10, except for their significantly lower RMSEV values: \pm 0.66 day for the fingermarks stored in the light, \pm 0.72 day in the dark and 0.94 day for the combination of the two storage conditions. In the light, 9 and 7-day old fingermarks showed good separation from the younger ones, but overlapped with each other (Figure 11A). In the dark (Figure 11B) and when combining both storage conditions (Figure 11C), 9-day old fingermarks could not be separated from 7-day old fingermarks but were differentiable from the younger ones, while 7-day old fingermarks could be separated from 1-day old but not from 3-day old fingermarks. The separation of 7 and 9-day old fingermarks from the younger ones was not possible when the model included 20 and 34-day old fingermarks.

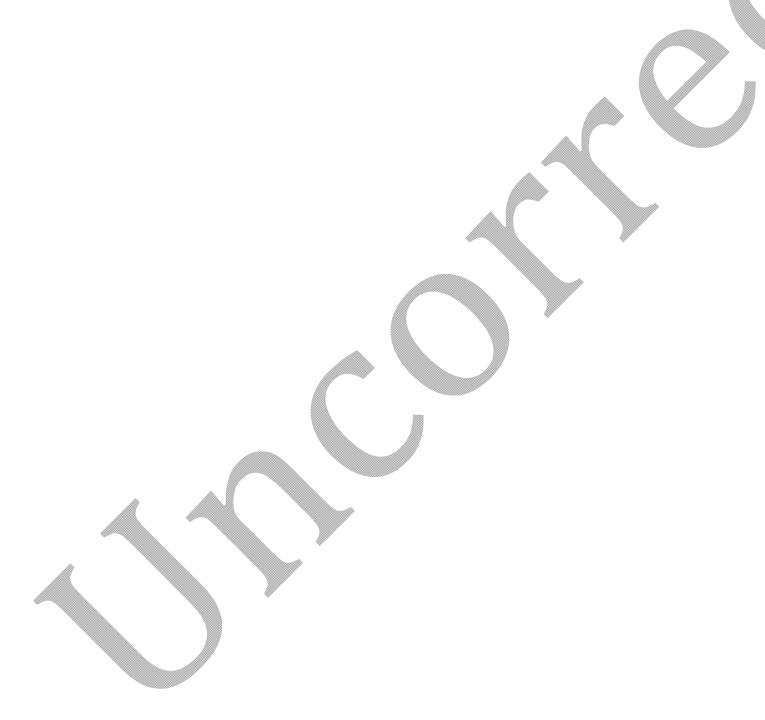

\footnotetext{
${ }^{1}$ RMSE indicates the quality of the regression fit; the lower the RMSE value, the better the model. While it can be considered as the global model error, the prediction error is recalculated for each new sample based on standard x-error calculations for linear regressions [69].
} 

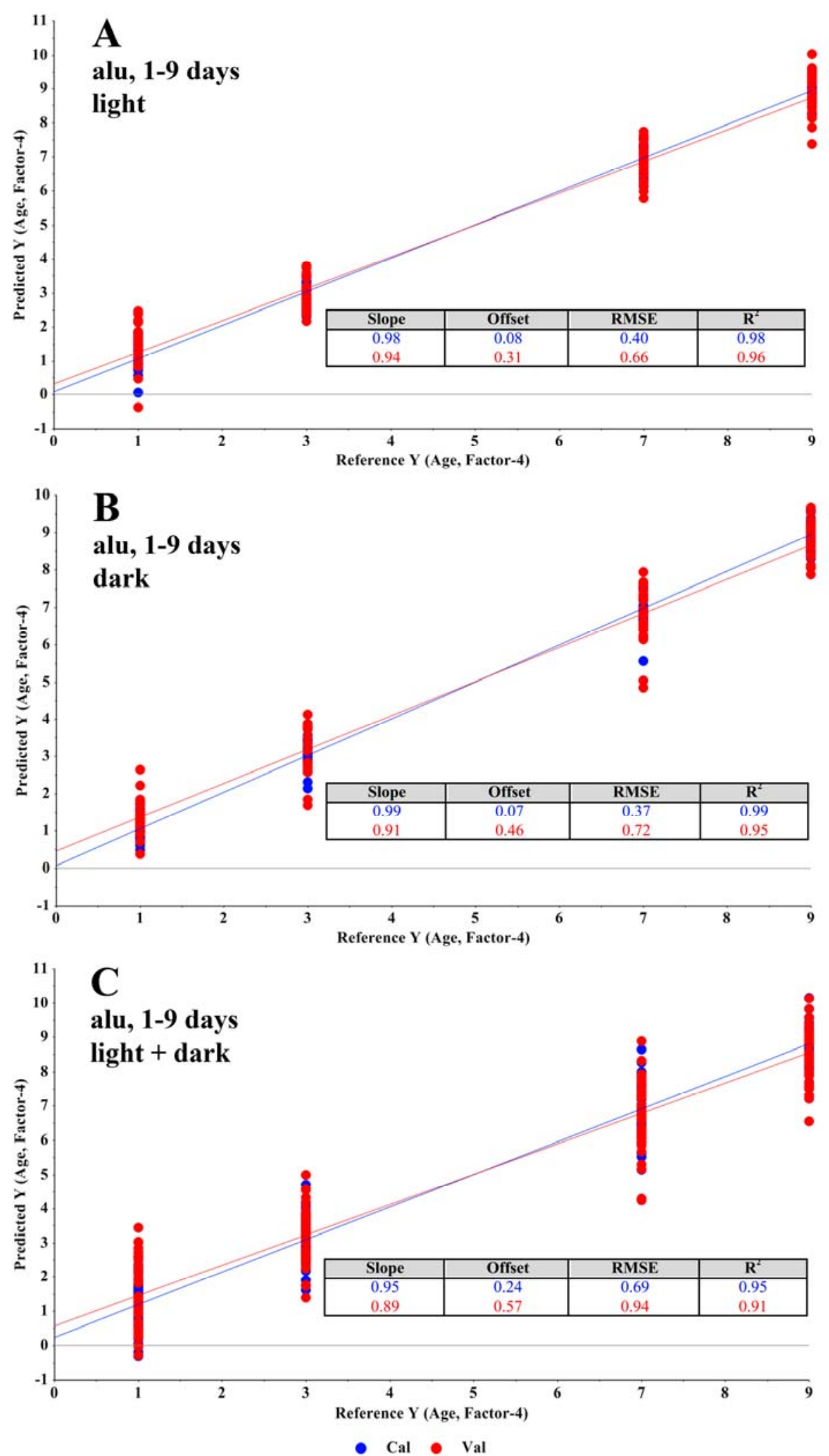

Figure 11 - Aging on aluminium from 1 to 9 days: PLS regression models obtained using fingermark spectra from 1 to 9-days old deposited on aluminium, acquired with ATR and stored A) in the light, B) in the dark and C) in the light or in the dark. Calibration (Cal, blue) and cross-validation (Val, red) points are shown. RMSE: root mean square error, $\mathrm{R}^{2}$ : coefficient of determination. 
PLS regressions were also conducted on fingermarks deposited on glass for 1, 3, 7 and 9 days and the results were obtained were similar to those for fingermarks deposited on aluminium during the same time period (see Figure 6 in the supplementary data).

\section{General Discussion and Conclusions}

In this work, the initial composition of fingermarks of one donor was studied using $\mu$-FTIR. The spectral regions $1000-1850 \mathrm{~cm}^{-1}$ and $2700-3600 \mathrm{~cm}^{-1}$ were identified as being the most informative and corresponded to molecular vibrations of eccrine and sebaceous material. Reflection and ATR modes were compared and ATR was selected for further analyses (substrate effects and aging) as it yielded better-resolved spectra on both aluminium and glass.

No significant differences due to the substrate were observed in fresh fingermarks. With respect to fingermark aging, it was observed that vibrational bands corresponding to eccrine material quickly disappeared over time, while sebaceous material decreased without completely disappearing over a period of up to 34 days. Using Spearman correlation calculations and PCA, it was also noted that fingermark spectra were correlated and could be grouped according to their age. However, influences of the substrate (i.e. aluminium vs. glass in this study) and storage conditions (i.e. storage in the light and in the dark in this study) on fingermark aging were noted. In fact, while no significant impacts were visually observed on the spectra, Spearman correlation calculations and PCA showed that the substrate had an effect on the aging of fingermarks when they were stored in the dark, but not in the light.

These observations are particularly important from a fingermark dating perspective. In fact, in practice, fingermark aging could be modelled based on the comparison of fingermarks from one suspect and taking into account known influence factors (e.g. substrate). However, these models should be as robust as possible to influence factors that cannot be precisely known (e.g. storage conditions). Thus, in this study, PLSR analyses encompassed fingermarks aged in the dark or in the light, but were conducted separately for each substrate. While the model precision decreased when both storage conditions were combined, it was still possible to differentiate 20 and 34-day old fingermarks from younger ones and a model precision of \pm 3 days was obtained. It was also noted that, when excluding the older fingermarks from the models (20 and 34-day old), the overall model precision increased to \pm 1 day, allowing the separation of 9 and 7-day old fingermarks from 1-day old specimens. Similar results were obtained for fingermarks deposited on aluminium and glass.

PLS regression models based on $\mu$-FTIR analyses should be further investigated for fingermark dating applications. Other influence factors should be considered and larger independent datasets should be collected in order to conduct full validation procedures using independent fingermark specimens. Furthermore, FTIR cannot be used on every type of surface, as it works best on non-porous substrates. Thus, the potential of PLSR to model aging of fingermarks left on porous surfaces could also be tested using data acquired with other analytical techniques, such as GC/MS.

\section{$\underline{\text { Acknowledgments }}$}

The authors wish to thank Cyril Muehlethaler (from the Ecole des Sciences Criminelles of Lausanne) for very fruitful discussions about chemometrics applied to FTIR data.

This work has been kindly supported by the Swiss National Science Foundation (SNF, fund no. PP00P1_123358/1). 


\section{$\underline{\text { References }}$}

[1] Cuthbertson F. (1969). The chemistry of fingerprints. United Kingdom Atomic Energy Authority, Atomic Weapons Research Establishment (AWRE). Report no. 013/69.

[2] Buchanan MV, Asano K, Bohanon A. (1996). Chemical characterization of fingerprints from adults and children. Forensic Evidence Analysis and Crime Scene Investigation, SPIE (International Society for Optical Engineering). 2941; 89-95.

[3] Mong GM, Petersen CE, Clauss TRW. (1999). Advanced Fingerprint Analysis Project Final Report - Fingerprint Constituents. Pacific Northwest National Laboratory.

[4] Archer NE, Charles Y, Elliott JA, Jickells S. (2005). Changes in the lipid composition of latent fingerprint residue with time after deposition on a surface. Forensic Science International. 154:(2-3); 224-39.

[5] Croxton RS, Baron MG, Butler D, Kent T, Sears VG. (2006). Development of a GC-MS method for the simultaneous analysis of latent fingerprint components. Journal of Forensic Sciences. 51:(6); 1329-33.

[6] Croxton RS, Baron MG, Butler D, Kent T, Sears VG. (2010). Variation in amino acid and lipid composition of latent fingerprints. Forensic Science International. 199:(1-3); 93-102.

[7] Richmond-Aylor A, Bell S, Callery P, Morris K. (2007). Thermal Degradation Analysis of Amino Acids in Fingerprint Residue by Pyrolysis GC-MS to Develop New Latent Fingerprint Developing Reagents. Journal of Forensic Sciences. 52:(2); 380-2.

[8] Koenig A, Girod A, Weyermann C. (2011). Identification of wax esters in fingermark residues by GC/MS and their potential use as aging parameters. Journal of Forensic Identification. 61:(6); 652-76.

[9] Weyermann C, Roux C, Champod C. (2011). Initial Results on the Composition of Fingerprints and its Evolution as a Function of Time by GC/MS Analysis. Journal of Forensic Sciences. 56:(1); 102-8.

[10] Mink T, Voorhaar A, Stoel R, de Puit M. (2013). Determination of efficacy of fingermark enhancement reagents; the use of propyl chloroformate for the derivatization of fingerprint amino acids extracted from paper. Science and Justice. 53:(3); 301-8.

[11] Girod A, Weyermann C. (2014). Lipid composition of fingermark residue and donor classification using GC/MS. Forensic Science International. 238; 68-82.

[12] Mountfort KA, Bronstein H, Archer N, Jickells SM. (2007). Identification of oxidation products of squalene in solution and in latent fingerprints by ESI-MS and LC/APCI-MS. Analytical Chemistry. 79:(7); 2650-7.

[13] Ifa DR, Manicke NE, Dill AL, Cooks RG. (2008). Latent fingerprint chemical imaging by mass spectrometry. Science. 321:(5890); 805.

[14] Wolstenholme R, Bradshaw R, Clench MR, Francese S. (2009). Study of latent fingermarks by matrix-assisted laser desorption/ionisation mass spectrometry imaging of endogenous lipids. Rapid Communication in Mass Spectrometry. 23; 3031-9.

[15] Tang HW, Lu W, Che CM, Ng KM. (2010). Gold nanoparticles and imaging mass spectrometry: Double imaging of latent fingerprints. Analytical Chemistry. 82:(5); 1589-93.

[16] Lim AY, Mab Z, Ma J, Rowell F. (2011). Separation of fingerprint constituents using magnetic silica nanoparticles and direct on-particle SALDI-TOF-mass spectrometry. Journal of Chromatography B. 879; 2244-50.

[17] Frick AA, Berryman D, Lewis SW. (2011). Mass spectral imaging: a powerful new tool for the study of latent fingermark chemistry. Identification Canada. September. 
[18] Emerson B, Gidden J, Lay JO, Durham B. (2011). Laser Desorption/onization Time-ofFlight Mass Spectrometry of Triacylglycerols and Other Components in Fingermark Samples. Journal of Forensic Sciences. 56:(2); 381-9

[19] Bailey MJ, Bright NJ, Croxton RS, Francese S, Ferguson LS, Hinder S, et al. (2012). Chemical characterization of latent fingerprints by matrix-assisted laser desorption ionization, time-of-flight secondary ion mass spectrometry, mega electron volt secondary mass spectrometry, gas chromatography/mass spectrometry, X-ray photoelectron spectroscopy, and attenuated total reflection Fourier transform infrared spectroscopic imaging: An intercomparison. Analytical Chemistry. 84:(20); 8514-23.

[20] West M, Went M. (2009). The spectroscopic detection of drugs of abuse in fingerprints after development with powders and recovery with adhesive lifters. Spectrochimica Acta Part A: Molecular and Biomolecular Spectroscopy. 71; 1984-8.

[21] West MJ, Went MJ. (2008). The spectroscopic detection of exogenous material in fingerprints after development with powders and recovery with adhesive lifters. Forensic Science International. 174:(1); 1-5.

[22] Emmons ED, Tripathi A, Guicheteau JA, Christesen SD, Fountain 3rd AW. (2009). Raman chemical imaging of explosive-contaminated fingerprints. Applied Spectroscopy. 63:(11); 1197-203.

[23] Connatser RM, Prokes SM, Glembocki OJ, Schuler RL, Gardner CW, Lewis SA, et al. (2010). Toward surface-enhanced raman imaging of latent fingerprints. Journal of Forensic Sciences. 55:(6); 1462-70.

[24] Tripathi A, Emmons ED, Guicheteau JA, Christesen SD, Wilcox PG, Emge DK, et al. (2010). Trace explosive detection in fingerprints with Raman chemical imaging. Proceedings of SPIE - The International Society for Optical Engineering, Orlando (FL), USA

[25] Tripathi A, Emmons ED, Wilcox PG, Guicheteau JA, Emge DK, Christesen SD, et al. (2011). Semi-Automated detection of trace explosives in fingerprints on strongly interfering surfaces with raman chemical imaging. Applied Spectroscopy. 65:(6); 611-9.

[26] Batrick E, Schwartz, R., Bhargava, R., Schaeberle, M., Fernandez, D., Levin, I. (2002). Spetrochemical analysis and hyperspectral imaging of latent fingerprints. 16th Meeting of the International Association of Forensic Sciences. Montpellier - France.

[27] Williams DK, Schwartz RL, Bartick EG. (2004). Analysis of Latent Fingerprint Deposits by Infrared Microspectroscopy. Applied Spectroscopy. 58:(3); 313-6.

[28] Wilkinson TJ, Martin, M.C., McKinney, W. R., Perry, D.L. (2004). Application of FTIR spectromicroscopy to the analysis of latent human fingerprints. 22th National Meeting of the American Chemical Society. Anaheim - Canada.

[29] Tahtouh M, Kalman, J. R., Roux, C., Lennard, C., Reedy, B. J. (2005). The detection and enhancement of latent fingermarks using infrared chemical imaging. Journal of Forensic Sciences. 50:(1); 64-72.

[30] Tahtouh M, Despland, P., Shimmon, R., Kalman, J. R., Reedy, B. J. (2007). The application of infrared chemical imaging to the detection and enhancement of latent fingerprints: Method optimization and further findings. Journal of Forensic Sciences. 52:(5); 1089-96.

[31] Tahtouh M, Scott SA, Kalman JR, Reedy BJ. (2011). Four novel alkyl 2-cyanoacylate monomers and their use in latent fingermark detection by mid-infrared spectral imaging. Forensic Science International. 207:(1-3); 223-38.

[32] Chan KLA, Kazarian SG. (2006). Detection of trace materials with Fourier transform infrared spectroscopy using a multi-channel detector. The Analyst. 131; 126-31. 
[33] Ricci C, Chan KLA, Kazarian SG. (2006). Combining the tape-lift method and fourier transform infrared spectroscopic imaging for forensic applications. Applied Spectroscopy. 60:(9); 1013-21.

[34] Ricci C, Bleay S, Kazarian SG. (2007). Spectroscopic imaging of latent fingermarks collected with the aid of a gelatin tape. Analytical Chemistry. 79:(15); 5771-6.

[35] Crane NJ, Bartick EG, Perlman RS, Huffman S. (2007). Infrared spectroscopic imaging for noninvasive detection of latent fingerprints. Journal of Forensic Sciences. 52:(1); 48-53.

[36] Ricci C, Phiriyavityopas P, Curum N, Chan KLA, Jickells S, Kazarian SG. (2007). Chemical imaging of latent fingerprint residues. Applied Spectroscopy. 61:(5); 514-22.

[37] Hemmila A, McGill, J., Ritter, D. (2008). Fourier transform infrared reflectance spectra of latent fingerprints: a biometric gauge for the age of an individual. Journal of Forensic Sciences. 53:(2); 369-76.

[38] Mou Y, Rabalais JW. (2009). Detection and Identification of Explosive Particles in Fingerprints Using Attenuated Total Reflection-Fourier Transform Infrared Spectromicroscopy. Journal of Forensic Sciences. 54:(4); 846-50.

[39] Chen T, Schultz ZD, Levin IW. (2009). Infrared spectroscopic imaging of latent fingerprints and associated forensic evidence. The Analyst. 134:(9), 1902-4.

[40] Ng PH, Walker S, Tahtouh M, Reedy B. (2009). Detection of illicit substances in fingerprints by infrared spectral imaging. Analytical and Bioanalytical Chemistry. 394:(8); 2039-48.

[41] Antoine KM, Mortazavi S, Miller AD, Miller LM. (2010). Chemical Differences Are Observed in Children's Versus Adults' Latent Fingerprints as a Function of Time. Journal of Forensic Sciences. 55:(2); 513-8.

[42] Abdelhamid M, Fortes FJ, Harith MA, Laserna JJ. (2011). Analysis of explosive residues in human fingerprints using optical catapulting-laser-induced breakdown spectroscopy. Journal of Analytical Atomic Spectrometry. 26:(7); 1445-50.

[43] Williams DK, Brown CJ, Bruker J. (2011). Characterization of children's latent fingerprint residues by infrared microspectroscopy: Forensic implications. Forensic Science International. 206:(1-3); 161-5

[44] De Grazia A, Mikhael M, Stojanovska N, Reedy B, Shimmon R, Tahtouh M. (2012). Diacetylene copolymers for fingermark development. Forensic Science International. 216:(13); 189-97.

[45] Fritz P, van Bronswjik W, Lepkova K, Lewis SW, Lim KF, Martin DE, et al. (2013). Infrared microscopy studies of the chemical composition of latent fingermark residues. Microchemical Journal. 111:(0); 40-6.

[46] Bright NJ, Willson, T.R., Driscoll, D.J., Reddy, S.M., Webb, R.P., Bleay, S., Ward, N.I., Kirkby, K.J., Bailey, M.J. (2013). Chemical changes exhibited by latent fingerprints after exposure to vacuum conditions. Forensic Science International. 230:(1-3); 81-6.

[47] Banas A, Banas K, Breese MBH, Loke J, Lim SK. (2014). Spectroscopic detection of exogenous materials in latent fingerprints treated with powders and lifted off with adhesive tapes. Analytical and Bioanalytical Chemistry. 406:(17); 4173-81.

[48] Olsen RD. (1987). Chemical Dating Techniques for Latent Fingerprints: A Preliminary Report. The Identification News. 10-2.

[49] De Puit M, Ismail M, Xu X. (2014). LCMS Analysis of Fingerprints, the Amino Acid Profile of 20 Donors. Journal of Forensic Sciences. 59:(2); 364-70.

[50] Ramotowski RS. (2001). Composition of Latent Print Residues in Advances in Fingerprint Technology: H.C. Lee, R.E. Gaensslen, Boca Raton, CRC Press. 63-104. 
[51] Champod C, Lennard C, Margot P, Stoilovic M. (2004). Fingerprints and other ridge skin impressions. 1st ed: CRC Press.

[52] Girod A, Ramotowski R, Weyermann C. (2012). Composition of fingermark residue: a qualitative and quantitative review. Forensic Science International. 223:(1-3); 10-24.

[53] Tahtouh M. (2008). Reagents for infrared chemical imaging of fingerprints on difficult surfaces: PhD Thesis, University of Technology of Sydney, Australia.

[54] Ricci C, Chan KLA, Kazarian SG. (2006). Fourier transform infrared spectroscopic imaging for the identification of concealed drug residue particles and fingerprints. Presented at the Optics and Photonics for Counterterrorism and Crime Fighting II Conference. Stockholm, Sweden.

[55] Ricci C, Kazarian SG. (2006). Enhancing forensic science with spectroscopic imaging. Presented at the Optics and Photonics for Counterterrorism and Crime Fighting II Conference, Stockholm.

[56] Ricci C, Kazarian, S. G. (2010). Collection and detection of latent fingermarks contaminated with cosmetics on nonporous and porous surfaces. Surface and Interface Analysis. 42:(5); 386-92.

[57] Adams MJ. (2004). Chemometrics in analytical spectroscopy. 2nd ed: RSC Analytical Spectroscopy Monographs.

[58] Geladi P, Sethson B, Nyström J, Lillhonga T, Lestander T, Burger J. (2004). Chemometrics in spectroscopy: Part 2. Examples. Spectrochimica Acta - Part B Atomic Spectroscopy. 59:(9); 1347-57.

[59] Muehlethaler C, Massonnet G, Esseiva P. (2011). The application of chemometrics on Infrared and Raman spectra as a tool for the forensic analysis of paints. Forensic Science International. 209:(1-3); 173-82.

[60] Rohman A, Man YBC. (2012). The chemometrics approach applied to FTIR spectral data for the analysis of rice bran oil in extra virgin olive oil. Chemometrics and Intelligent Laboratory Systems. 110:(1); 129-34.

[61] Girod A, Ramotowski R, Lambrechts S, Misrielal P, Aalders M, Weyermann C. (2015). Fingermark dating: legal considerations, review of the literature and practical propositions. Forensic Science International. (to be submitted).

[62] Girod A, Roux C, Weyermann C. (2014). La datation des traces digitales (partie II): proposition d'une approche formelle. Revue internationale de criminologie et de police technique. 68:(2); 226-49.

[63] Stuart BH. (2005). Infrared Spectroscopy: Fundamentals and Applications: John Wiley \& Sons, Ltd, Chichester, UK.

[64] Pavia DL, Lampman GM, Kriz GS, Vyvyan JR. (2009). Introduction to spectroscopy: A guide for students of organic chemistry. 3rd ed: 4th edition, Brooks/Cole, Belmont (USA).

[65] Smith BC. (2011). Fundamentals of Fourier Transform Infrared Spectroscopy, Second Edition: CRC Press.

[66] Rinnan A, van den Berg F, Balling Engelsen S. (2009). Review of the most common preprocessing techniques for near-infrared spectra. Trends in Analytical Chemistry. 28:(10); 1201-22.

[67] Jolliffe IT. (2002). Principal Component Analysis (Second Edition): Springer Series in Statistics.

[68] Vandeginste BGM, Massart, D.L., Buydens, L.M.C., De Jong, S., Lewi, P.J., SmeyersVerbeke, J. (1997). Data handling in science and technology 20B: Handbook of chemometrics and qualimetrics, Part B. Amsterdam: Elsevier. 
[69] Mecklin CJ, Mundfrom DJ. (2004). An Appraisal and Bibliography of Tests for Multivariate Normality. International Statistical Review. 72:(1); 123-38.

[70] Farrell PJ, Salibian-Barrera M, Nackz K. (2006). On tests for multivariate normality and associated simulation studies. Journal of Statistical Computation and Simulation. 00:(00); 114.

[71] Bartlett AA. (2004). The Essential Exponential: For the Future of Our Planet: University of Nebraska Press: Lincoln, NE.

[72] Lociciro S, Dujourdy L, Mazzella W, Margot P, Lock E. (2004). Dynamic of the ageing of ballpoint pen inks: quantification of phenoxyethanol by GC-MS. Science and Justice. 44:(3); 165-71.

[73] Weyermann C, Kirsch, D., Costa Vera, C., Spengler, B. (2007). A GC/MS study of the drying of ballpoint pen ink on paper. Forensic Science International. 168:(2-3); 119-27.

[74] Gallidabino M, Weyermann C, Romolo FS, Taroni F. (2013). Estimating the time since discharge of spent cartridges: A logical approach for interpreting the evidence. Science and Justice. 53:(1); 41-8.

[75] Sheskin DJ. (2004). Handbook of Parametric and Nonparametric Statistical Procedures. Third Edition ed. Chapman \& Hall / CRC.

[76] Hauke J, Kossowski T. (2011). Comparaison of values of Pearson's and Spearmann's correlation coefficients on the same sets of data Questiones Geographicae. 30:(2); 87-93.

[77] Esbensen KH, Guyot D, Westad F, Houmøller LP. (2002). Multivariate data analysis in practice: an introduction to multivariate data analysis and experimental design: 5th Edition, Camo Process AS, Oslo.

[78] Ramer G, Lendl B. (2013). Attenuated Total Reflection Fourier Transform Infrared Spectroscopy in Encyclopedia of Analytical Chemistry: John Wiley \& Sons, Ltd. 